\title{
Nanomechanical and microstructual properties of binary and ternary blended cement paste containing sustainable materials
}

\author{
J. Chakkamalayath*, S. Al-Bahar and A. Joseph \\ Construction and Building Materials Program, Energy and Building Research Center, \\ Kuwait Institute for Scientific Research, Safat 13109, Kuwait \\ *Email: jchakkamalayath@kisr.edu.kw
}

Extreme hot and cold climatic conditions along with the marine environment creates a very aggressive atmosphere affecting the durability and serviceability of concrete structures in Kuwait and the Gulf region. Nanoscience along with nano engineering has helped to characterize the materials in the micro and nano scale, for developing innovative construction materials as well as for improving the macro scale applications of conventional materials for sustainable infrastructure suitable to these aggressive climates. Also, binary and ternary blended concrete containing nano materials and other supplementary cementitious materials has become increasingly important because of their increased durability, economic and environmental advantages.

The nano-mechanical properties of cementitious materials were studied using nanoindentation, a unique technique that has been developed to improve the macro mechanical performance of concrete in terms of strength development, tailoring of desired properties and durability [1]. The application of this test method is also extended to study the effect of different mineral admixtures on the properties of blended cement pastes on a micro and nano scale level. The basic principles of nanoindentation test and the results of preliminary investigation to understand the effect of supplementary cementitious materials on the nano mechanical properties of cement paste is presented in this paper. In this study, the images of indentations at different phases were also obtained along with the stiffness and hardness values using loading and unloading data as shown in Figure 1.

Further to this, the mean modulus and hardness values obtained from the nanoindenation test was used to identify the different phases in the hardened cement paste, although additional tests are required to confirm this.
The incorporation of nanomaterials on the modification to the microstructure of the hydrated cement paste after 28 days of curing is also discussed in this paper. Binary and ternary blended cementitious systems based on ordinary Portland cement with volcanic ash and nanosilica/microsilica were prepared. Sacnning Electron Microscope (SEM) analysis [2] shows a significant amount of hydration reaction after 28 days of curing and a much denser gel morphological structure was observed for samples containing nanosilica than microsilica as shown in Figure 2.

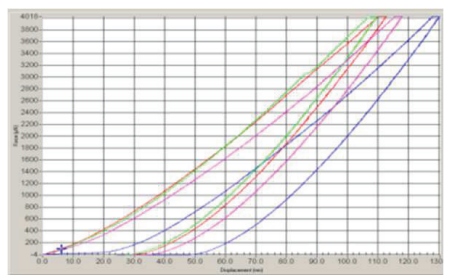

Figure 1: Loading and unloading curve in nanoindentation test

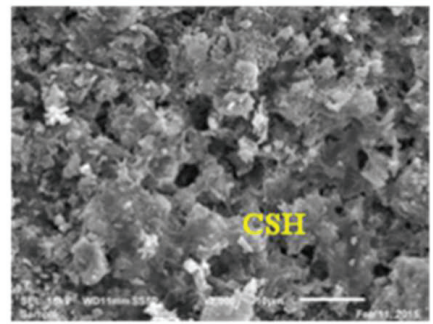

Figure 2: SEM image of ternary blend with volcanic ash and nanosilica

\section{References}

1. Oliver, W.C. and G.M. Pharr. 1992. Journal of Materials Research 7: 1564-1583.

2. Elena, J. and M. D. Lucia. 2012. Journal of Applied Engineering Sciences, 2: 35-42. 